\title{
FACTORES NUTRICIONALES Y ALTERACIÓN EN EL CONTENIDO DE ANTOCIANINAS RELACIONADOS CON EL ENNEGRECIMIENTO DE LOS PÉTALOS EN ROSA (Rosa sp.)
}

\section{NUTRITIONAL FACTORS AND MODIFICATION IN THE CONTENT OF ANTHOCYANINS ASSOCIATED WITH THE BLACKENING OF ROSE PETALS (Rosa sp.)}

\begin{abstract}
Wilson Bohórquez ${ }^{1}$, John Gómez ${ }^{2}$, Víctor Flórez ${ }^{3}$
${ }^{1}$ Microbiólogo Agrícola, Especialista en Horticultura, Magíster en Ciencias Agrarias, Facultad de Agronomía, Universidad Nacional de Colombia, sede Bogotá. wbohorquezs@gmail.com; ${ }^{2}$ Ingeniero Agrónomo, Esp. Estadística Aplicada, profesor Facultad Ciencia y Tecnología U.D.C.A. johgomez@udca.edu.co; ${ }^{3}$ Doctor en Ciencias, profesor Facultad de Agronomía, Universidad Nacional de Colombia, sede Bogotá. Cra. 30 No. 45-03, Ciudad Universitaria, Bogotá, D.C., Colombia. Autor de Correspondencia: vjflorezr@unal.edu.co
\end{abstract}

Rev. U.D.C.A Act. \& Div. Cient. 16(1): 103 - 112, 2013

\section{RESUMEN}

Uno de los desórdenes fisiológicos más importantes en los cultivares de rosa roja corresponde al ennegrecimiento de los pétalos, lo que afecta pétalos externos e internos y resulta en flores de baja calidad. Esta revisión de literatura tiene por objetivo abordar la fisiopatía del ennegrecimiento de los pétalos, desde sus causas más posibles, con la finalidad de poner a disposición de productores, de académicos y de científicos, así como de los interesados en general, insumos que permitan desvendar su origen y optimizar su manejo. Los síntomas del ennegrecimiento de los pétalos se manifiestan, especialmente, cuando las flores son cosechadas y almacenadas en condiciones de cuarto frío, debido a la acumulación de pigmentos del tipo de las antocianinas, posiblemente, a causa de condiciones de estrés, como alta o baja radiación UV, bajas temperaturas o a un desbalance de nutrientes en los tejidos afectados. Se profundizará sobre: i) los nutrientes calcio y boro y su interacción, ii) el calcio y los desórdenes fisiológicos, iii) los pigmentos involucrados en el color rojo de los pétalos y iv) el papel de los compuestos fenólicos, en particular de las antocianinas, las cuales, son las responsables de la mayoria de los colores rojo, rosado, purpura y azul observados en las partes de la planta; así como, sobre factores ambientales que, en su conjunto, pudieran influir en la fisiopatía del ennegrecimiento de los pétalos de la rosa roja.

Palabras clave: Fenoles, antocianinas, interacción calcio boro.

\section{SUMMARY}

One of the most important physiological disorders of red rose cultivars is blackening of petals, it affects outer and inner ones and results in flowers of poor quality. The goal of this review is to make an approach to the blackening disorder, determining the main possible causes in order to make available to growers, academics, scientifics as well as stakeholders, the inputs that allows to unveil its origin and to optimize its handling. Symptoms of blackening are showed especially when the flowers have been harvested and stored under cold room conditions; due to high accumulation of pigments, such as anthocyanins, also could be due to stress conditions, such as high or low UV radiation, low temperatures or a nutrient imbalance in the affected tissues. The following subjects will be treated: i) calcium and boron and their interaction, ii) calcium and physiological disorders, iii) pigments involved in the petals red color, iv) role of the phenolic compounds, particularly the anthocyanins, which are responsible for most of the red, pink, purple and blue colors observed in plant parts; as well as the environmental factors that can influence the blackening on red rose.

Key words: Phenolics, anthocyanins, calcium and boron interaction.

\section{INTRODUCCIÓN}

El manejo agronómico y los factores ambientales imperan- 
tes en la precosecha inciden, directamente, sobre diferentes procesos bioquímicos y fisiológicos y pueden afectar parámetros de calidad en las flores de corte.

Uno de los problemas en poscosecha que disminuye el valor de ornato en rosas rojas es el ennegrecimiento de los pétalos, que consiste en una necrosis de los ápices de los pétalos, que puede extenderse a la totalidad de la flor. A pesar que se han realizado diferentes investigaciones con el propósito de dilucidar sus causas, aún no existen aspectos concluyentes sobre el tema. Entre las causas sugeridas, se encuentran condiciones ambientales, como bajas temperaturas, alta radiación lumínica y bajos niveles de calcio y de boro (Cabrera et al. 2007); sin embargo, los resultados obtenidos en diferentes investigaciones con respecto al papel de elementos nutritivos en el fenómeno del ennegrecimiento son contradictorios. Para Franck et al. (2007) es ocasionado por una acumulación de compuestos fenólicos, en particular pigmentos del tipo antocianinas, debido a diferentes causas aún no determinadas. En la mayoría de las ocasiones los síntomas aparecen después que la flor es cortada.

Con el propósito de indagar sobre la participación de factores nutricionales y la alteración en la concentración de pigmentos del tipo de antocianinas en el fenómeno del ennegrecimiento de los pétalos de rosa, se hizo la presente revisión de literatura. Este estudio permitirá a productores, académicos y científicos, así como a los interesados en general, abordar dicha fisiopatía, desde sus causas más posibles y, de esta forma, optimizar su manejo.

\section{Factores nutricionales en el ennegrecimiento de los pé-} talos:

El calcio: El calcio $\left(\mathrm{Ca}^{++}\right)$es relativamente inmóvil en el floema de la planta, lo cual, significa que una vez ubicado en un tejido en particular, su movimiento es lento o casi nulo (Epstein, 1973). Esto sugiere que los órganos vegetales con crecimiento activo, tales como los frutos carnosos, los bulbos y las flores necesitan un suministro constante del elemento. Según Reuter \& Robinson (1986), el requerimiento de calcio para Rosa spp. está entre 1 a 1,5\%. Su transporte a larga distancia, generalmente, es por el xilema (espacios intercelulares: apoplasto) o por medio de los plasmodesmos (simplasto). En el apoplasto, parte del $\mathrm{Ca}^{++}$está firmemente ligado a las estructuras, otra parte es intercambiable en las paredes celulares y en la superficie exterior de la membrana plasmática. Una elevada cantidad de $\mathrm{Ca}^{++}$forma complejos con aniones orgánicos, como el malato e inorgánicos, como el nitrato y el cloruro y se almacenan en las vacuolas, cloroplasto y retículo endoplásmico rugoso, mientras que su concentración en el citoplasma es extremadamente baja $\left(10^{-7} \mathrm{M}\right)$. Una concentración baja de $\mathrm{Ca}^{++}$en el citoplasma es esencial para prevenir la precipitación del $\mathrm{Pi}$, competencia con el magnesio por los sitios de unión y, posiblemente, como prerrequisito para que funcione como mensajero secundario (Marschner, 1995).

Respecto a su función como mensajero secundario, al percibir la planta un estímulo externo, se presenta una entrada de $\mathrm{Ca}^{++}$al citoplasma desde los sitios de almacenamiento. Esto puede activar varias enzimas, a través de la fosforilación dependiente de proteínas quinasas, que están involucradas en diferentes reacciones metabólicas, que conducen a respuestas en los procesos de crecimiento y desarrollo vegetal (Poovaiah, 1988).

La mayoría de las funciones del $\mathrm{Ca}^{++}$como componente estructural de las membranas y paredes celulares, se debe, principalmente, a la capacidad que tiene el elemento para formar complejos estables, pero reversibles con los pectatos de la lamela media, que están conformadas de cadenas de residuos de ácido poligalacturónico con inserciones de ramnosa, de grupos fosfatos y de carboxilatos de las proteínas, manteniendo, de esta manera, la permeabilidad selectiva, la integridad y la compartimentalización celular (Blowers \& Trewavas, 1988; Roux \& Slocum, 1982; Rahman \& Punja, 2007).

Debido a que su transporte se realiza, preferiblemente, por las células muertas del xilema, los síntomas visuales de deficiencia, por lo general, son observados en los tejidos jóvenes. Las zonas meristemáticas de las raíces, los tallos y las hojas, donde existen divisiones celulares permanentes, son las más susceptibles, quizás porque es fundamental para que se forme una nueva lamela media (Taiz \& Zeiger, 2006).

Frecuentemente, es observado que los órganos de las plantas que presentan baja transpiración manifiestan desórdenes, debido a deficiencia de $\mathrm{Ca}^{++}$. Las rosas de corte, así como los frutos y los tubérculos, se encuentran dentro de esta categoría. El $\mathrm{Ca}^{++}$, en dichos órganos, es conducido dentro de la planta, generalmente, por el xilema (Marschner, 1995) y, por lo tanto, la baja transpiración podría resultar en menor cantidad del elemento transportado hacia esos órganos. Este fenómeno ha sido propuesto para explicar por qué las rosas crecidas en alta humedad relativa presentan menores concentraciones del elemento y menor vida en florero (Torre et al. 1999).

Una baja concentración de $\mathrm{Ca}^{++}$en los tejidos que muestra baja tasa de transpiración es necesaria para permitir rápida expansión celular y elevada permeabilidad en sus membranas celulares; sin embargo, se incrementa el riesgo que su contenido en los tejidos caiga por debajo del nivel crítico requerido, para la estabilización de la pared celular y la integridad de la membrana y, quizá también, para que funcione como mensajero secundario (Taiz \& Zeiger, 2006).

Aunque los cambios fisiológicos y bioquímicos de la senes- 
cencia están controlados genéticamente, existe evidencia que supone la participación del $\mathrm{Ca}^{++}$en este proceso. Su función estaría ligada al mantenimiento estructural de algunas moléculas de la membrana celular, a través de uniones con moléculas cargadas negativamente, como los fosfolípidos y las proteínas, que mantienen funcionando a la célula (Blowers \& Trewavas, 1988; Roux \& Slocum, 1982). Itzhaki et al. (1998) y Torre et al. (1999) reportaron que el $\mathrm{Ca}^{++}$ retrasa la descomposición de tales moléculas en el proceso senescente. De igual manera, Borochov et al. (1982) mencioan que en la senescencia de pétalos de rosa se disminuye la fluidez de las membranas celulares, debido a una pérdida de los fosfolípidos y a la disminución en su síntesis.

La deficiencia de $\mathrm{Ca}^{++}$, especialmente en el tejido meristemático, puede reducir la estabilidad de la pared y la membrana celular, conduciendo a la muerte del tejido. Su deficiencia puede ser debida más a un transporte inadecuado por los conductos vasculares de la planta que causada por baja absorción o disponibilidad en el suelo (Cresswell \& Weir, 1997).

Es indispensable separar los fenómenos de "deficiencia" y de "estrés" de $\mathrm{Ca}^{++}$, para entender lo relacionado con la dinámica de este elemento en la fisiología de la planta. Una "deficiencia" se logra corregir, a través de la adición de una fuente del elemento, mientras que el "estrés" es causado por una localización inadecuada del elemento; en este caso, los síntomas no se expresan en la totalidad de la planta, sino en puntos específicos de crecimiento del tejido (Cresswell \& Weir, 1997).

El estrés abiótico, frecuentemente, conduce a un incremento del $\mathrm{Ca}^{++}$libre en el citoplasma de las células, que conlleva a la expresión génica que activa respuestas bioquímicas, que permiten a la planta adaptarse a condiciones adversas de diferente naturaleza. De esta manera, el $\mathrm{Ca}^{++}$está involucrado en los mecanismos regulatorios, que le facultan a la planta realizar ajustes en condiciones adversas, como temperatura alta, daño por frío y estrés hídrico y salino (Liang et al. 2009). Esto ha conducido a relacionar las deficiencias del elemento en los tejidos de la planta con la aparición de desórdenes fisiológicos presentados en diferentes cultivos, especialmente en tejidos y en órganos, que presentan una baja tasa de transpiración (Martyn et al. 2007).

Es así como Collier \& Huntington (1983) y Martyn et al. (2007) relacionan la deficiencia de $\mathrm{Ca}^{++}$en los tejidos directamente con desórdenes fisiológicos, como la quemazón del ápice en lechuga, pardeamiento en Telopea spp. y en Euphorbia pulcherrima, pudrición apical en tomate y ennegrecimiento de pétalos de rosa; no obstante, Murray et al. (1972) investigaron estos desórdenes fisiológicos en tomate y en flores de corte sin encontrar diferencias significativas en la concentración del $\mathrm{Ca}^{++}$en los tejidos y en los órganos afectados, en comparación con los que no expresaron los síntomas característicos. Al respecto, Franco et al. (1999) observaron una alta incidencia de la pudrición apical en tomate, pese a encontrar niveles altos de $\mathrm{Ca}^{++}$en la parte distal del fruto. Adicionalmente, Chiu \& Bould (1976) no observaron la manifestación del desorden en plantas crecidas con bajo suministro de $\mathrm{Ca}^{++}$.

Debido a lo anterior, Wissemeier (1993), Saure (1998) y Franco et al. (1999) cuestionan el papel del $\mathrm{Ca}^{++}$como causa primaria y fundamental en estos desórdenes y sugieren que, por encima de su concentración en los tejidos, factores ambientales, como temperaturas extremas, transpiración alta, viento y radiación lumínica alta, pueden estar más comprometidos con la manifestación de los síntomas.

Por ejemplo, al estudiar el ennegrecimiento de brácteas de Telopea spp., Martyn et al. (2007) no encontraron relación entre las brácteas afectadas por este síntoma y la concentración de $\mathrm{Ca}^{++}$en los tejidos. Además, las brácteas de las plantas cultivadas bajo sombra mostraron menor afectación que las sometidas a luz plena, lo que demuestra que la radicación lumínica de alguna forma está relacionada con el desorden. Lo que indica que las condiciones ambientales que conducen la planta a situaciones de estrés podrían influir en la manifestación de los síntomas. Por otra parte, la incidencia de otro desorden fisiológico, conocido como quemazón del ápice, que afecta a lechuga, coliflor y calabacín, se reduce significativamente, cuando las plantas se someten a condiciones de baja radiación lumínica (Wissemeier, 1996).

Las condiciones ambientales que conducen al estrés resultan más críticas, cuando los tejidos susceptibles se encuentran en estadios de máxima tasa de división y de crecimiento celular (Saure, 2001 y 1998; Palzkill et al. 1980). Por ejemplo, una mayor incidencia en el pardeamiento de las brácteas de Telopea spp. coincidió con la etapa de máximo desarrollo de sus inflorescencias (Martyn et al. 2007).

Por otra parte, el crecimiento rápido de los tejidos vegetales estaría en función de una reducción en la concentración de $\mathrm{Ca}^{++}$que, a su vez, estaría asociado a un incremento en la actividad de las giberelinas, que interfieren en la toma y transporte del $\mathrm{Ca}^{++}$en los tejidos de las plantas (Grunzweig et al. 2000); mientras el $\mathrm{Ca}^{++}$protege la integridad de las membranas celulares, reduciendo su permeabilidad y previniendo la liberación de iones, a causa del estrés (Grattan \& Grieve, 1999), las giberelinas tienen el efecto opuesto, por lo tanto, la susceptibilidad al estrés se incrementa por bajas concentraciones de $\mathrm{Ca}^{++} \mathrm{y}$ altos niveles de giberelinas (Fletcher et al. 2000).

De esta forma, las bajas concentraciones de $\mathrm{Ca}^{++}$presentes en los tejidos en activo crecimiento pueden ser fisiológica- 
mente razonables, tal como lo señala Marschner (1983), al indicar que en los tejidos en rápido crecimiento se requiere una alta permeabilidad de la membrana para el flujo de los solutos. Esto significa, que en estos tejidos una alta demanda en el suministro de $\mathrm{Ca}^{++}$podría no existir, ya que, incluso, se reduciría el crecimiento de brotes y de frutos y la producción total (Hadi et al. 1996).

A pesar de los resultados contradictorios en cuanto al papel de los factores externos y endógenos en estos desórdenes, es evidente que son promovidos por situaciones de estrés, que resultan más severos cuanto más intenso es el estrés y más susceptible el cultivar (Saure, 1998).

Si la hipótesis es correcta al indicar que en los diferentes desórdenes fisiológicos vinculados, inicialmente con deficiencia de $\mathrm{Ca}^{++}$en los tejidos, los síntomas del daño y la baja tolerancia, debidos al estrés resultan en niveles elevados de giberelinas activas, entonces, la deficiencia localizada de $\mathrm{Ca}^{++}$ sería un indicador primario del incremento de la actividad de esta clase de hormonas, más que la causa fundamental del problema (Saure, 1998).

El papel del $\mathrm{Ca}^{++}$aplicado consistiría, principalmente, en contrarrestar el efecto de las giberelinas sobre las membranas celulares, pero su efectividad para controlar la incidencia del desorden dependería del nivel de estrés: si el crecimiento es vigoroso y no es seguido por estrés severo, no se esperaría mayor incidencia, independientemente de la cantidad del elemento aplicado (Saure, 1998).

El Boro: El boro (B) está implicado en la síntesis, en el mantenimiento y en la estabilidad de la pared celular; en el metabolismo de carbohidratos, RNA, auxinas y fenoles y en la respiración y transporte, a través de membrana (Bolaños et al. 2004; Cara et al. 2002), por lo tanto, su deficiencia, puede causar daños irreversibles a las plantas, cuando se encuentran en estadios fenológicos críticos (Lehto et al. 2004).

El requerimiento de $B$, se encuentra en un rango de 2 en monocotiledóneas hasta $80 \mathrm{mg} \cdot \mathrm{L}^{-1}$ en dicotiledóneas. La cantidad óptima para algunas especies puede ser tóxica o insuficiente para otras (Karabal et al. 2003). Para el caso de Rosa spp., la concentración se encuentra entre 30 y $60 \mathrm{mg}^{-1} \mathrm{~L}^{-1}$ (Reuter \& Robinson, 1986).

Las raíces de las plantas absorben $\mathrm{B}$ como ácido bórico no disociado $\mathrm{H}_{3} \mathrm{BO}_{3}$ y borato, transportados en las hojas, vía xilema. La transpiración es un factor clave que afecta su absorción y su concentración en los tejidos, aunque también es móvil por el floema y puede ser retranslocado en cantidades considerables, por lo tanto, la reducción en el transporte de agua puede reducir la acumulación de niveles tóxicos en los tejidos (Karabal et al. 2003).
El B se encuentra en las células formando complejo con las pectinas y con los fenoles y en forma libre (Ruiz et al. 1998). Es un elemento esencial para la estructura de la pared celular, debido a la formación de complejos con pectina, a través de uniones ester con grupos hidroxilos de carbohidratos o glucoproteínas (Pfeffer et al. 2001). Según Blevins \& Lukaszewski (1998), en condiciones de deficiencia de B, se interrumpe la síntesis de la pared celular.

El ácido bórico tiene la capacidad de formar complejos con moléculas que contienen grupos dioles y polioles, especialmente cis-dioles, dentro de su estructura. Dentro de tales compuestos, se incluyen diferentes azúcares y sus derivados (azúcares alcoholes y ácidos urónicos), en especial manitol y sorbitol. Estos compuestos funcionan como constituyentes de la fracción de hemicelulosa de las paredes celulares (Blevins \& Lukaszewski, 1998). En contraste, azúcares, como la glucosa, la fructosa, la galactosa y sus derivados (sacarosa, por ejemplo), no contienen la configuración cis-diol y, por lo tanto, no forman complejos estables con el borato (Marschner, 1995). La proporción relativa del B unido a la pared celular varía con la especie, órgano y suministro de boro (Ishii \& Matsunaga, 2001).

Las uniones que el borato forma con los componentes de la pared celular son relativamente débiles y, por consiguiente, apropiadas para permitir el rompimiento y la reconstrucción, durante la elongación celular. Adicionalmente, provee cargas negativas para las interacciones iónicas necesarias para el crecimiento celular (Marschner, 1995).

En estudios in vivo e in vitro realizados por Bolaños et al. (2004), se comprobó que el $\mathrm{Ca}^{++}$inhibe la descomposición del dímero RGII-B (Ramnogalacturona II -B) de la pared celular. Con base en trabajos sobre la estabilidad de diferentes fracciones de B en raíces intactas, Apostol \& Zwiazek (2004) sugieren que el $\mathrm{Ca}^{++}$puede incrementar la fortaleza de los complejos RGII-B. Además, reportan alteraciones en la traslocación de $\mathrm{Ca}^{++}$en brotes y en frutos en plantas que presentan deficiencia de B.

Por otra parte, la acumulación de fenoles libres es una característica de las plantas deficientes en B y probablemente se relaciona con la función que realiza el microelemento, mediante la formación de complejos cis - dioles con determinados azúcares y fenoles. Al presentarse dicha deficiencia, el flujo de sustratos se direcciona hacia el ciclo de las pentosas fosfato, aumentando, de esta manera, la biosíntesis de fenoles. La formación de estos complejos, quizá está involucrada en la regulación de fenoles libres (Cara et al. 2002). En deficiencia de $\mathrm{B}$, los fenoles se acumulan en los tejidos y la actividad de la polifenol oxidasa se incrementa, conduciendo a la producción de quinonas en las paredes celulares. Estas moléculas son efectivas en la producción de radicales supe- 
róxido, potencialmente eficaces en el daño a las membranas celulares, a través de la peroxidación de los lípidos (Marschner, 1995).

De igual manera, la radiación lumínica alta conduce a mayores requerimientos de $\mathrm{B}$. El requerimiento adicional de $\mathrm{B}$ es utilizado para detoxificar o neutralizar las mayores concentraciones de fenoles, a través de la formación de los complejos borato - fenol (Marschner, 1995). Así, se ha propuesto que la pérdida en la integridad de la membrana, causada por niveles deficientes de $\mathrm{B}$ puede ser debido a una acumulación de productos fenólicos y sus productos oxidados, los cuales, causan alteraciones en dicha estructura celular (Cara et al. 2002).

Las Antocianinas: Son compuestos fenólicos del grupo de los flavonoides, que se encuentran principalmente en frutos, en flores y en hojas (Lee et al. 2011), responsables de conferir los colores rojo, azul y violeta (Leguizamón et al. 2005; Tatsuzawa et al. 2010), dependiendo de la copigmentación o interacción con compuestos, como metales, alcaloides, taninos y polisacáridos (Lee et al. 2008). Por ejemplo, la copigmentación entre antocianinas y flavonoles en los pétalos de las flores de Heliophila coronopifolia, da como resultado su coloración azul (Saito et al. 2011). Las antocianinas también tienen un papel importante en la ecología de las plantas, ya que al completarse el desarrollo del fruto, su presencia transforma su color a rojo o púrpura, lo que conduce a la dispersión de las semillas por parte de pájaros y otros animales (Petroni \& Tonelli, 2011).

Las antocianinas, se caracterizan por su alto poder reductor y pueden ser inducidas rápidamente en respuesta a condiciones de estrés, ocasionados por factores abióticos o bióticos (Petroni \& Tonelli, 2011); por ejemplo, alta radiación lumínica , bajas temperaturas, deficiencias de elementos minerales como nitrógeno, fósforo, boro y manganeso (Whittaker et al. 2009) o incidencia de patógenos (Jakopic et al. 2009; Leguizamón et al. 2005; Grotewold, 2006), condiciones que conllevan a la producción exagerada de especies reactivas de oxígeno. De esta manera, las antocianinas pueden neutralizar los efectos nocivos de los radicales libres que causan daño y muerte celular o disipar energía radiante, que no puede ser utilizada en el proceso fotosintético ( $\mathrm{O}^{\prime}$ Neill $\&$ Gould, 2003). Solamente, seis antocianinas son comunes en los vegetales superiores: cianidina, pelargonidina, peonidina, malvidina, petunidina y delfinidina (Lee et al. 2005), siendo cianidina 3-glucósido la antocianina con mayor poder antioxidante (Grotewold, 2006) y la de mayor contenido en diferentes cultivares de rosa (Tatsuzawa et al. 2010; Lee et al. 2011).

La biosíntesis de antocianinas, se lleva a cabo a partir de la ruta del ácido siquímico y su regulación se realiza de manera diferente en monocotiledóneas y dicotiledóneas (Petroni \&
Tonelli, 2011); se acumulan, especialmente, en las vacuolas de las células epidérmicas y mesofílicas (Schmitzer et al. 2009). Su estructura molecular puede variar, debido a la sustitución glucosílica que presenten (glucosa, galactosa, rhamnosa, xylosa y arabinosa) y a la acilación de los grupos de azúcares con ácidos, en especial, ácido acético, cumárico, caféico, malónico, felúrico, oxálico y succínico (Grotewold, 2006).

La naturaleza y el número de azúcares ligados a la molécula, la posición de esta unión, la naturaleza y el número de ácidos alifáticos y aromáticos que se unen al azúcar durante la acetilación, juegan un rol importante en el color y en la estabilidad del compuesto. Otros factores de estabilización son la formación de complejos con iones metálicos, como aluminio e hierro (Markovic et al. 2005), presencia de proteínas, pH, luz y temperatura (Rodriguez-Saona \& Wrolstad, 2001; Kim et al. 2011; Schmitzer et al. 2012).

En cuanto al papel del pH, Brouillard (1988) estableció que el valor de este parámetro en la vacuola de la célula puede afectar el color de los pétalos, a través de la alteración física entre los electrones del pigmento. Por otra parte, en Hydrangeas sp., cultivadas en suelo con el pH de 5,5, el color de la flor es azul, pero si el pH incrementa a 6,0, el color es rosado. En este caso, a pH bajo, el aluminio se torna más disponible y se encuentra en altas concentraciones en los pétalos, formando complejo con las antocianinas que resultan en el color azul (Quintana et al. 2007).

Alteración en el Contenido de Antocianinas: En la mayoría de las plantas, la biosíntesis y la acumulación de antocianinas en las flores, se encuentra bajo regulación ambiental y endógena y coincide con el crecimiento de los pétalos (Mol et al. 1996). Su acumulación es parte integral del desarrollo de las flores que, posiblemente, son reguladas por los mismos factores que controlan su crecimiento (Weiss, 2000). En cuanto a la biosíntesis presentada en los frutos, Chen et al. (2012) indican que en mora (Rubus spp. cv. Arapoho), la concentración de antocianinas es baja en los estados iniciales de desarrollo y se incrementa drásticamente cuando los frutos alcanzan la madurez.

De acuerdo con lo anterior, Halevy \& Zieslin (1974), investigando los factores ambientales y bioquímicos implicados en el ennegrecimiento de los pétalos de rosa cv. Baccara, encontraron que los pétalos de los tallos afectados contenían entre dos y cinco veces más antocianinas totales y la PPO (Polifenol oxidasa) presentaba una actividad significativamente más alta, en comparación con los pétalos de los tallos florales no afectados. Las flores afectadas por los síntomas fueron aquellas procedentes de plantas que crecieron fuera de los invernaderos, sometidas a bajas temperaturas nocturnas (Halevy \& Zieslin, 1974; Jaffrin, 2002). 
Por otro lado, trabajos realizados en vid, confirmaron que la alta radiación lumínica incidente en los racimos provocaron un incremento en la concentración total de antocianinas monoméricas y flavonoles, pero se redujeron por las excesivas temperaturas (Steyn et al. 2000). De igual forma, Iglesias et al. (1999) reportaron que el nivel de antocianinas en la epidermis de frutos de manzano estaba inversamente relacionado con la temperatura, especialmente, con la nocturna.

En cuanto al efecto que produce el estrés fisiológico sobre las antocianinas, Wanga et al. (2012) indican que en la epidermis de frutas de uvas, procedentes de plantas cultivadas bajo restricción radicular, se encontró una mayor concentración total e individual de estos pigmentos y que la acumulación de azúcares en los órganos reproductivos de las plantas sometidas a este tipo de estrés, estaría relacionado con el aumento considerable de las antocianinas.

De igual manera, la luz induce la acumulación de antocianinas en tejidos verdes y en células en suspension: plantas crecidas con baja intensidad lumínica desarrollan flores pálidas con bajo nivel de estos pigmentos (Griesbach, 1992). En los tejidos verdes, las antocianinas actúan como pigmentos de protección, que absorben la luz UV. La radiación UV-B (280 a 320nm) induce la síntesis de antocianinas en las hojas de varias especies de plantas (Jenkins et al. 1995); sin embargo, el efecto que tiene la radiación UV sobre la pigmentación de los pétalos los y frutos, por lo tanto, en la síntesis de antocianinas depende de la especie vegetal, ya que en algunas es requerida y, en otras, no tiene ningún efecto (Weiss, 2000; Petroni \& Tonelli, 2011). Por ejemplo, en hojas de diferentes líneas de maíz híbrido, la radiación UV-B incrementa significativamente el contenido de antocianinas, posiblemente, en respuesta metabólica al estrés ocasionado. De esta manera, las plantas sembradas en los lugares con mayor nivel de radiación presentaron contenidos de antocianinas $45 \%$ mayores en comparación con los cultivares que crecieron con menor radiación. La mayor concentración de antocianinas es una respuesta de las plantas a los efectos adversos de este tipo de radiación, los cuales, pueden causar mortalidad del polen, asincronia en floración y esterilidad en plantas de maíz (Pinter et al. 2007).

De acuerdo con Lu et al. (2009), cerca del 52\% de la variación del contenido de antocianinas en pétalos de diferentes cultivares de Ipomoea purpurea puede ser explicado por el efecto de la temperatura y la radiación UV. Así, las plantas cultivadas, tanto en campo como en invernadero, aumentaron la concentración de antocianinas, cuando estuvieron expuestas a bajas temperaturas y alta radiación lumínica.

Ennegrecimiento de los pétalos: El ennegrecimiento de pétalos de rosa, se manifiesta como áreas negras o cafés hundidas en los ápices de los pétalos, síntomas frecuentemente evidentes después de la cosecha, lo que en ocasiones da lugar a flores severamente afectadas.

Algunos cultivadores de rosas en Colombia muestran que la fisiopatía se desarrolla especialmente en días nublados, en donde prevalecen temperatura baja y alta humedad relativa. Además, manifiestan que existe un fuerte componente genético involucrado, ya que algunos cultivares son más susceptibles que otros. Los síntomas más evidentes en los cultivares susceptibles, se suelen presentar cuando los tallos florales son sometidos al tratamiento de frío habitual, en la producción comercial (Cabrera et al. 2007).

De acuerdo con Cabrera et al. (2007), en los cultivos comerciales de rosa de cultivares susceptibles en California (Estados Unidos), el desorden se manifiesta cuando se presentan temperaturas entre 10 y $15^{\circ} \mathrm{C}$ por una a dos semanas antes que la flor sea cosechada. El mantenimiento de la temperatura por encima de $15^{\circ} \mathrm{C}$, conlleva a la supresión de los síntomas.

En trabajo de investigación llevado a cabo recientemente por los autores de la presente revisión, la privacion de calcio y de boro en la solución nutritiva de plantas de rosa cv. Freedom, afectó la acumulación de estos elementos en los tejidos, lo que confirma el sinergismo de estos dos elementos; sin embargo, el contenido de pigmentos de tipo antocianinas no mostró diferencias significativas entre los tratamientos. Se infiere que las condiciones ambientales presentadas durante el desarrollo de las plantas pudo tener una implicación más directa en el ennegrecimiento de los pétalos que el balance nutricional.

Desórdenes similares al ennegrecimiento de los pétalos de rosa, se presentan en hortalizas (lechuga, tomate, calabacín), frutos (manzano y peral) y en ornamentales, posiblemente, debido a una deficiencia de $\mathrm{Ca}^{++}$, aunque diferentes investigaciones indican que las causas podrían estar más relacionadas con factores ambientales que conduce al estrés (Franck et al. 2007; Wissemeier, 1993; Saure, 1998).

En lo que respecta al papel de la parte nutricional en el desorden, experimentos realizados con rosa roja cv. Royalty, mostraron que la incidencia del ennegrecimiento en los pétalos fue generalmente mayor en las plantas que se cultivaron en soluciones nutritivas que contenían bajas concentraciones de $\mathrm{Ca}^{++}$y B y que al aumentar la concentración de estos elementos, los síntomas disminuyeron; no obstante, sorprendió que los tallos cosechados dos días después del punto comercial de corte no presentaban los síntomas, sin importar la solución nutritiva utilizada. Se infiere que existe un estadio en el desarrollo de la flor, en el cual, se presenta una mayor susceptibilidad en la expresión del desorden, en condiciones que promueven una baja absorción y acumula- 
ción de estos minerales, especialmente, en hojas y en tallos (Cabrera et al. 2007).

Lo anterior confirma que las concentraciones bajas de $\mathrm{Ca}^{++}$ por sí mismas no son la causa fundamental del problema, excepto en las condiciones de estrés, producidas en el proceso de poscosecha de la flor cortada.

Un desorden fisiológico relacionado con el ennegrecimiento de los pétalos de rosa es el pardeamiento de algunos frutales, como el peral y el manzano, que se presenta por oxidaciones enzimáticas de compuestos fenólicos, debido a la acción de la polifenol oxidasa (PPO) a quinonas, moléculas muy reactivas, que forman polímeros de color marrón. Los factores más importantes involucrados en el pardeamiento enzimático son la concentración de fenoles y la actividad de la PPO (Franck et al. 2007). Al respecto, Hamauzu \& Hanakawa (2003) encontraron que las variedades de peral que evidenciaron menor concentración de compuestos fenólicos fueron menos susceptibles al pardeamiento.

De acuerdo con los resultados previamente reportados sobre las causas del ennegrecimiento de los pétalos en rosas rojas (Halevy \& Zieslin, 1974; Cabrera et al. 2007), es posible inferir que la predisposición genética del cultivar y las bajas temperaturas son los factores que más influyen en la incidencia de este desorden fisiológico. Es probable, que las condiciones nutricionales y los portainjertos utilizados para el desarrollo de las plantas de rosa (Rosa sp.), se puedan considerar factores secundarios.

Conflictos de intereses. El manuscrito fue preparado y revisado con la participación de todos los autores, quienes declaramos que no existe ningún conflicto de intereses que ponga en riesgo la validez de los resultados presentados. Financiación. Este estudio fue financiado por la División de Investigación de la Universidad Nacional de Colombia, sede Bogotá, en el marco del proyecto «Papel de la interacción calcio $\mathrm{x}$ boro en el desarrollo floral en el ennegrecimiento de los pétalos de rosa».

\section{BIBLIOGRAFÍA}

1. APOSTOL, K.; ZWIAZEK, J. 2004. Boron and water uptake in jack pine (Pinus Banksiana) seedlings. Environ. Exp. Bot. 51(2):145-153.

2. BLOWERS, D.P.; TREWAVAS, A.J. 1988. Phosphatidylinositol kinase activity of a plasma membraneassociated calcium-activated protein kinase from pea. Fed. Eur. Biochem. Soc. 238(1):87-89.

3. BLEVINS, D.; LUKASZEWSKI, K. 1998. Boron in plant structure and function. Ann. Rev. Plant Physiol. 49:481-500.

4. BOLAÑOS, L.; LUKASZEWSKI, K.; BONILLA, I.; BLEVINS, D. 2004. Why boron? Plant Physiol. Biochem. 42(11):907-912.

5. BOROCHOV, A.; MAYAK, S.; BROUN, R. 1982. The involvement of water stresses and ethylene in senescence of carnation flowers. J. Exp. Bot. 33:1202-1209.

6. BROUILLARD, R. 1988. The Flavonoids: Advances in research. In: Harborne, J.B. (Ed.). Flavonoids and flower color. Chapman \& Hall, (London). p.525-538.

7. CABRERA, R.I.; SOLÍS-PÉREZ, A.R.; McCORMICK, J. 2007. The role of calcium and boron in rose development and petal blackening: Observations in commercial rose greenhouses and shoot tissue nutrient status. Progress Report (July to December). Texas A\&M University. Research and Extension Center. 5p.

8. CARA, F.; SÁNCHEZ, E.; RUIZ, J.; ROMERO, L. 2002. Is phenol oxidation responsible for the short term effect of boron deficiency on plasma-membrane permeability and function in squash root? Plant Physiol. Biochem. 40(10):853-858.

9. CHEN, Q.; YU, H.; TAN, H.; WANG, X. 2012. Identification and expression analysis of genes involved in anthocyanin and proanthocyanidin biosynthesis in the fruit of blackberry. Sci Hort. 141:61-68.

10. CHIU, T.F.; BOULD, C. 1976. Effects of shortage of calcium and other cation on Ca mobility, growth and nutritional disorders of tomato plants (Lycopersicon esculentum). J. Sci. Food Agr. 27:969-977.

11. COLLIER, G.F.; HUNTINGTON, V.C. 1983. The relationship between leaf growth, calcium accumulation and distribution, and tipburn development in field grown butterhead lettuce. Sci. Hortic. 21(2):123-128.

12. CRESSWELL, G.C.; WEIR, R.G. 1997. Plant Nutrient Disorders 5: Ornamental Plants and Shrubs. Inkata Press, Melbourne. 234p.

13. EPSTEIN, E. 1973. Flow in the phloem and the immobility of calcium and boron: A new hypothesis in support of an old one. Experienta. 29:133-136.

14. FLETCHER, R.; GILLEY, A.; SANKHLA, N.; DAVIS, T. 2000. Triazoles as plant growth regulator and stress protectants. Hort. Rev. 24:55-138. 
15. FRANCO, J.A.; PEREZ-SAURA, P.J.; FERNANDEZ, J.A.; PARRA, M.; GARCIA, A.L. 1999. Effect of two irrigation rates on yield, incidence of blossom-end rot, mineral content, and free amino acid levels in tomato cultivated under drip irrigation using saline water. J. Hort. Sci. \& Biotechn. 74:430-435.

16. FRANCK, C.; LAMMERTYN, J.; HO, Q.T.; VERBOVEN, P.; VERLINDEN, B.E.; NICOLAI, B.A. 2007. Review: Browning disorders in pear fruit. Posth. Biol. Techn. 43:1-13.

17. GRATTAN. S.; GRIEVE, C. 1999. Salinity-mineral nutrient relations in horticultural crops. Sci. Hort. 78:127-157.

18. GRIESBACH, R.J. 1992. Correlation of $\mathrm{pH}$ and light intensity on flower color in potted Eustoma grandiflorum Grise. Hort. Sci. 27(7):817-818.

19. GROTEWOLD, E. 2006. The genetics and biochemistry of floral pigments. Ann. Rev. Plant Biol. 57:761-780.

20. GRUNZWEIG, J.; RABINOWICH, H.; KATAN J.; WODNER, M.; BEN - TAL, Y. 2000. Involvement of endogenous gibberellins in the regulation of increased tomato shoot growth in solarized soil. Plant Growth Reg. 30:233-239.

21. HADI, M.; CONWAY, W.; SAM, C. 1996. Effect of calcium on yield and incidence of blossom-end rot of three tomato cultivars. J. Hort. Sci. 31:672-679.

22. HALEVY, A.; ZIESLIN, N. 1974. The development and causs of petal blackening and malformation of "Baccara" rose flower. Acta Hort. 14:149-155.

23. HAMAUZU, Y.; HANAKAWA, T. 2003. Relation of highly polymerised procyanidin to the potential browning susceptibility in pear fruits. J. Jap. Soc. Hort. Sci. 72:415-421.

24. IGLESIAS, J.; GRAELI, G.; ECHEVERRIA, M.; VENDRELL. 1999. Differences in fruit color development, anthocyanin content, yield and quality of seven delicious apple strains. Fruit Var. J. 53(1):133-145.

25. ISHII, T.; MATSUINAGA, T. 2001. Pectic polysaccharide rhamnogalacturonan II is covalently linked to homogalacturonan Phytochem. 57:969-974.

26. ITZHAKI, H.; NAVEH, L.; LINDAHL, M.; COOK, M.; ADAM, Z. 1998. Identification and characterization of DegP, a serine protease associated with the lume- nal side of the thylakoid membrane. J. Biol. Chem. 273:7094-7098.

27. JAFFRIN, A. 2002. Ennegrecimiento de los pétalos de rosas bajo diferentes tipos de invernadero. Plasticulture. 121(3):12-21.

28. JAKOPIC, J.; STAMPAR, F.; VEBERIC, R. 2009.The influence of exposure to light on the phenolic content of 'Fuji' apple. Sci. Hort. 123:234-239.

29. JENKINS, G.I.; CHRISTIE, J.M.; FUGLEVAND, G.; LONG, J.C.; JACKSON, J.A. 1995. Plant responses to UV and blue light: Biochemical and genetic approaches. Plant Sci. 112:117-138.

30. KARABAL, E.; YÜCEL, M.; AVNI, H. 2003. Antioxidant responses of tolerant and sensitive barley cultivars to boron toxicity. Plant Sci. 164 (6):925-933.

31. KIM, D.; BAE, J.; LEE. D.; LEE, H.; JOO, M.; YOO, S. 2011. Positive effects of glycosylated anthocyanin isolated from an edible berry fruit (Acanthopanax sessiliflorum) on its antioxidant activity and color stability. Food Res. Int. 44:2258-2263.

32. LEE, J.; LEE, H.; CHOUNG, M. 2011. Anthocyanin compositions and biological activities from the red petals of Korean edible rose (Rosa hybrida cv. Noblered). Food Chem. 129:272-278

33. LEE, J., RENNAKER, C.; WROLSTAD, R.E. 2008. Correlation of two anthocyanin quantification methods: HPLC and spectrophotometric methods. Food Chem. 110:782-786.

34. LEE, J.; DURST, R.W.; WROLSTAD, R.E. 2005. Determination of total monomeric anthocyanin pigment content of fruit juices, beverages, natural colorants, and wines by the $\mathrm{pH}$ differential method: Collaborative study. J. AOAC Int. 88(5):1269-1278.

35. LEGUIZAMÓN, G.V.; GONZALEZ, L.A.; BAEZ, S.R. 2005. Grape anthocyanins (Vitis vinifera L.) and their relation to color. Rev. Fitotec. Mex. 28(4):359368.

36. LEHTO, T.; RÄISÄNEN, M.; LAVOLA, A.; JULKUNENTITTO, R.; APHALO, P. 2004. Boron movility in deciduos forest trees in relation to their polyls. New Phytol. 163:333-339.

37. LIANG, W.J.; WANG, M.L.; AI, X.Z. 2009. The role of calcium in regulating photosynthesis and related phy- 
siological indexes of cucumber seedlings under low light intensity and suboptimal temperature stress. Sci. Hort. 123:34-38.

38. LU, Y.; DU, J.; TANG, J.; WANG, F.; ZHANG, J.; HUANG, J.; LIANG, W.; WANG, L. 2009. Environmental regulation of floral anthocyanin synthesis in Ipomoea purpurea. Molec. Ecol. 18:3857-3871.

39. MARKOVIC, D.J.; NADEZJDA, J.; PETRANOVIC, A.; BARANAC, J.M. 2005. The copigmentation effect of sinapic acido on malvidin: a spectroscopic investigation on color enhancement. J. Photochem. Photobiol. B, Biol. 78(3):223-228.

40. MARSCHNER, H. 1995. Mineral nutrient of higher plants, 2nd. ed. Academic Press (London). 446p.

41. MARSCHNER, H. 1983. General introduction to the mineral nutrition of plants. In: Läuchli, A.; Bieleski, R.L. (eds.) Encyclopedia of Plant Physiology, New Series, Vol. 15A. Springer-Verlag (Berlin). p.5-60.

42. MARTYN, A.; THOMAS, CH.; O'NEILL, M.; OFFORD, C.; McCONCHIE, R. 2007. Bract browning in waratahs (Telopea spp.) is not a localized calcium deficiency disorder. Sci. Hort. 112:434-438.

43. MARTYN, A.; GOLLNOW, B.; McCONCHIE, R.; OFFORD, C. 2007. Characterization of bract browning and the effect of shade on browning in waratah (Telopea spp., Proteaceae) cultivars 'Fire and Brimstone', 'Olympic Flame' and 'Wirrimbirra White'. Sci. Hort. 112:427-433.

44. MOL, J.; JENKINS, G.; SCHAFER, E.; WEISS, D. 1996. Signal perception, transduction, and gene expression involved in anthocyanin biosynthesis. Crit. Rev. Plant Sci. 15:525-557.

45. MURRAY, S.A.; SPURR, A.R.; PAULSON, K.N. 1972. Electron probe analysis of $\mathrm{Ca}, \mathrm{Mg}$ and $\mathrm{K}$ in the fruit of the tomato, Lycopersicon esculentum, with reference to blossom-end rot. Agr. J. 64(5):586-588.

46. O'NEILL, S.; GOULD, S.K. 2003. Anthocyanis in leave: light attenuators or antioxidants? Func. Plant Biol. 30:865-873.

47. PALZKILL, D.A.; TIBBITTS, T.W.; STRUCKMEYER, B.E. 1980. High relative humidity promotes tipburn on young cabbage plants. HortSci. 15:659-660.
48. PETRONI, K.; TONELLI, Ch. 2011.Recent advances on the regulation of anthocyanin synthesis in reproductive organs. Ann. Rev. Plant Sci. 181:219-229

49. PFEFFER, H.; DANNEL, F.; RÖMHELD, V. 2001. Boron compartmentation in root sunflower: A study using the stable isotopes $10 \mathrm{~B}$ and $11 \mathrm{~B}$ adopting two independent approaches. Physiol. Plant. 113:346-351.

50. PINTER, J.; KÓSA, E.; HADI, G.; HÉGYI, Z.; SPITKO, T., TOTH, Z.; SZIGETI, Z.; PALDI, E.; MARTON, C. 2007. Effect of increased UV- radiation on the anthocyanin content of maize (Zea mays L) leaves. Acta Agr. Hung. 55(1):7-17.

51. POOVAIAH, B.W. 1988. The molecular and cellular aspects of calcium action. HortSci. 23:267-271.

52. QUINTANA, A.; JANA ALBRECHTOVA, J.; GRIESBACH, R.; FREYRE, F. 2007. Anatomical and biochemical studies of anthocyanidins in flowers of Anagallis monelli L. (Primulaceae) hybrids. Sci. Hort. 112:413-421.

53. RAHMAN, M.; PUINJA, Z. 2007. Calcium and plant disease. In: Datnoff, L.E.; Elmer, W.H., and Huber, D.M. (eds).Mineral Nutrition and Plant disease. The American Phytopathological Society. Ed. APS Press (St. Paul). 7993p.

54. REUTER, D.J.; ROBINSON, J.B. 1986. Plant Analysis: An Interpretation Manual. Ed. Inkata Press (Melbourne). 218p.

55. RODRIGUEZ-SAONA, L.E.; WROLSTAD, R.E. 2001. Extraction, isolation and purification of anthocyanins. Current Protocols in Food Analytical Chemistry. John Willey and Sons. Inc. 11p.

56. ROUX, S.J.; SLOCUM, R.D. 1982. Role of calcium in mediating cellular functions important for growth and development in higher plants. In: Cheung, W. Y. ed. Calcium and cell function, Vol III. Academic Press (New York), p.408-453.

57. RUIZ, J.; BRETONES, G.; BAGHOUR, M.; RAGALA, L.; BELAKBIR, A.; ROMERO, L. 1998. Relationship between boron and fenolic metabolism in tobacco leaves. Phytochem. 48(2):269-272.

58. SAITO, N.; TATSUZAWA, F.; TOKI, K.; SHINODA, K.; SHIGIHARA, A.; HONDA, T. 2011. The blue anthocyanin pigments from the blue flowers of Heliophila coronopifolia L. (Brassicaceae). Phytochem. 72:2219-2229. 
59. SAURE, M. 1998. Causes of the tipburn disorder in leaves of vegetables. Sci. Hort. 76:131-147.

60 SAURE, M. 2001. Blossom-end rot of tomato (Lycopersicon esculentum Mill.) a calcium - or a stress-related disorder? Sci. Hort. 90:193-208.

61. SCHMITZER, V.; OSTERC, G.; VEBERIC, R.; STAMPAR, F. 2009. Correlation between chromaticity values and major anthocyanins in seven Acer palmatum Thunb. cultivars. Sci. Hort. 119:442-446.

62. SCHMITZER, V.; VEBERIC, F.; STAMPAR, F. 2012. Prohexadione-Ca application modifies flavonoid composition and color characteristics of rose (Rosa hybrida L.) flowers. Sci Hort. 146:14-20.

63. STEYN, W.J.; HOLCROFT, D.M.; WAND, S.J.E.; COOKS, N.C.; JACOBS, G. 2000. Dating Rosemarie: How to make her blush?. Proceedings of the cape Pomolological association Symposium. South Africa. p.55-62.

64. TAIZ, L.; ZEIGER, E. 2006. Plant Physiology. $3^{\text {rd }}$ edition, Sinanuer Associate, Inc. (USA). 690p.

65 TATSUZAWA, F.; ICHIHARA, K.; SHINODA, K.; MIYOSHI, K. 2010. Flower colours and pigments in Disa hybrid (Orchidaceae). South Afr. J. Botany. 76:4953.

66. TORRE, S.; BOROCHOV, A.; HALEVY, A.H. 1999. Calcium regulation of senescence in rose petals. Phys. Plant. 107:214-219.
67. WANGA, B.; HEA, J.; DUANB, Ch.; YUA, X.; ZHUA, L.; XIEA, Z.; ZHANGA, C.; XUA, W.; WANG, S. 2012. Root restriction affects anthocyanin accumulation and composition in berry skin of 'Kyoho' grape (Vitis vinifera L. $\times$ Vitis labrusca L.) during ripening. Sci Hort. 137:20-28.

68. WISSEMEIER, A.H. 1996. Calcium-Mangel bei Salat (Lactuca sativaL.) und Poinsettie (Euphorbia pulcherrima Willd. ex Klotzsch): Einfluß von Genotyp und Umwelt. Verlag Ulrich E. Grauer (Stuttgart). 299p.

69. WISSEMEIER, A.H. 1993. Marginal bract necrosis in Poinsettia cultivars and the relationship to bract calcium nutrition. Gartenbauwiss. 58:158-163.

70. WEISS, D. 2000. Regulation of flower pigmentation and growth: Multiple signaling pathways control anthocyanin synthesis in expanding petals. Phys. Plant. 110:152-157.

71. WHITTAKER, A.; VAZZANA, C.; VECCHIO, V.; BENEDETTELLI, S. 2009. Evaluation of direct and indirect effects of flavonoids, mineral elements and dry weight on antiradical scavenging activity in leaf material of field-grown Trifolium pratense cultivars using Path Analysis. Field Crops Res. 113:1-11.

Recibido: Febrero 12 de 2012

Aceptado: Febrero 28 de 2013 